\title{
含咔唑基苯并咪唑衍生物的合成
}

\author{
六垒曾向潮* 何如罗创龙林志强
}

(暨南大学化学系 广州 510632)

\begin{abstract}
摘要 以咔唑为原料, 经烷基化、甲酰化反应合成 $N$-烃基咔唑-3-甲醛; 无催化剂条件下, $N$-烃基咔唑-3-甲醛与邻苯二 胺经缩合-环化 “一锅” 反应合成了两类新型含咔唑单元的苯并咪唑衍生物. 研究了溶剂选择、溶剂用量、反应温度、 反应物配比等因素对缩合一环化反应结果的影响; 通过改变两种反应物的等物质的量比可选择性地得到以其中一类苯 并咪唑衍生物为主的产物.

关键词 苯并咪唑; 咔唑; 有机合成
\end{abstract}

\section{Syntheses of Benzoimidazole Derivatives Containing Carbazole Unit}

\author{
Bian, Lei \\ Zeng, Xiangchao* \\ $\mathrm{He}, \mathrm{Ru}$ \\ Luo, Chuanglong \\ Lin, Zhiqiang \\ (Department of Chemistry, Jinan University, Guangzhou 510632)
}

\begin{abstract}
Two series of novel benzoimidazole derivatives containing carbazole unit were synthesized via condensation-cyclization of $\mathrm{N}$-alkylcarbazole-3-formaldehyde with 1,2-diaminobenzene in "one pot" reaction without catalyst. The effects of temperature, selection \& amount of solvents and molar ratio of the two reagents on the reactions have been investigated; significantly, one of these two series of benzoimidazole derivatives could be prepared as the major products selectively by changing the molar ratio of the two reagents.
\end{abstract}

Keywords benzoimidazole; carbazole; organic synthesis

苯并咪唑及其衍生物是一类重要的生物活性物质, 在药物领域具有广谱活性, 如抗肿瘤 ${ }^{[1]}$ 、抗 HIV-1 病

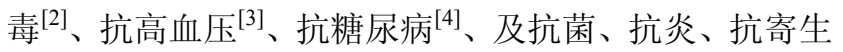
虫等 ${ }^{[5,6]}$. 同时，因具有优良的光学性能、丰富的 $\pi$ 电子、 良好的刚性平面结构和稳定性等优点, 苯并咪唑化合物 及其配合物被广泛用于分子/离子识别 ${ }^{[7,8]}$ 、光电材 料 ${ }^{[9-11]}$ 和金属有机催化剂 ${ }^{[12]}$ 等领域. 因此, 合成含苯并 咪唑结构化合物对新药及新材料的研究和开发都具有 十分重要的意义. 研究表明, 具有大范围共轭结构的电 子给体能有效提高光敏材料的发光性能 ${ }^{[9,13]}$, 咔唑是蓝 色磷光主体材料中常见的分子片段 ${ }^{[14]}$. 鉴于此, 本文选 用结构简单、具有优越空穴传输性能的咔唑化合物为原 料, 合成了具有拓展 $\pi$ 结构的目标化合物, 期待篮选和 进一步合成有更佳性能的光敏材料.

苯并咪唑衍生物的合成一般经两种途径: 第一种是 以邻苯二胺与羧酸或其衍生物为原料, 在盐酸 ${ }^{[15]}$ 、多聚 磷酸 ${ }^{[16]}$ 等作用下合成, 这种方法通常需要强酸性条件、
反应时间较长, 有些反应要在高温下进行. 第二种是近 年来报道较多的方法, 其以邻苯二胺与醛类化合物为原 料, 在氧化剂如 $\mathrm{DDQ}^{[17]}, \mathrm{Me}_{2} \mathrm{~S}^{+} \mathrm{BrBr}^{-[18]}$, 或催化剂如 $\mathrm{Co}(\mathrm{OH})_{2}$ 和 $\mathrm{CoO}^{[19]}, \mathrm{Ce}\left(\mathrm{NH}_{4}\right)_{2}\left(\mathrm{NO}_{3}\right)_{6}{ }^{[20]}$, 苯磺酸 ${ }^{[21]}$ 等作 用下经环化一氧化脱氢合成. 然而, 这种方法也有一些 不足之处，如后处理复杂、需化学计量甚至过量的氧化 剂，许多反应还产生毒性大或污染环境的副产物等. 本 文以咔唑为原料，经烷基化、甲酰化反应，合成 $N$-烃基 咔唑- 3-甲醛, 然后以无水甲醇为溶剂, 所制备的 $N$-烃 基咔唑- 3-甲醛与邻苯二胺在 $45{ }^{\circ} \mathrm{C}$ 、无催化剂存在下经 缩合-环化 “一锅” 反应合成了两类含咔唑结构的苯并 咪唑衍生物 2 和 3 (Scheme 1).

\section{1 结果与讨论}

Varala $^{[22] 、 \text { 杨红伟等 }}{ }^{[23]}$ 报道, 邻苯二胺与两倍量的 芳醛反应先生成双席夫碱中间产物，后者再重排得到 1,2-二取代苯并咪唑衍生物. Lin 等 ${ }^{[2]}$ 报道, 苯甲醛等与

*E-mail: xczeng@126.com

Received November 13, 2013; revised January 20, 2014; published online January 24, 2014.

Project supported by the University Student Innovative Program of Guangdong Province (No. 1055912003).

广东省大学生创新(No. 1055912003)资助项目. 
<smiles>[R]n1c2ccccc2c2cc(C=O)ccc21</smiles><smiles></smiles>

a: $\mathrm{R}=\mathrm{C}_{2} \mathrm{H}_{5} ; \mathbf{b}: \mathrm{R}=\left(\mathrm{CH}_{3}\right)_{2} \mathrm{CH} ; \mathbf{c}: \mathrm{R}=\mathrm{CH}_{2}=\mathrm{CHCH}_{2}$;

d: $\mathrm{R}=n-\mathrm{C}_{4} \mathrm{H}_{9} ; \mathbf{e}: \mathrm{R}=n-\mathrm{C}_{6} \mathrm{H}_{13}$

\section{Scheme 1}

邻苯二胺反应, 经单席夫碱关环生成苯并咪唑啉中间 体, 后者经空气中 $\mathrm{O}_{2}$ 氧化脱氢生成 2-取代苯并咪唑衍 生物. 崔丽君等 ${ }^{[25]}$ 报道, 4-甲基苯甲醛与邻苯二胺在等 物质的量的比为 $1: 1$ 时反应, 同时生成经单席夫碱或 双席夫碱中间体转化的两种苯并咪唑衍生物. 根据实验 结果, $N$-烃基咔唑-3-甲醛与邻苯二胺的反应也同时生成 两种苯并咪唑衍生物(可能的反应机理见 Scheme 2), 而 溶剂的选择及用量、反应物配比和反应温度等均对反应 结果有显著的影响(表 1).
溶剂的选择及用量对反应产物的组成和产率有较 大的影响. 以 $10 \mathrm{~mL}$ 无水甲醇为溶剂、 $2.2 \mathrm{mmol} N$-烯丙 基咔唑-3-甲醛与 $1.0 \mathrm{mmol}$ 邻苯二胺于 $45{ }^{\circ} \mathrm{C}$ 反应 $7 \mathrm{~h}$, 化合物 2c 产率为 78.6\%, 化合物 3c 产率为 18.5\%(表 1, Entry 1). 选用乙腈替代甲醇作溶剂进行反应，产物 $2 \mathrm{c}$ 的产率仅为 $35.7 \%$, 而 3c 的产率增大为 $36.3 \%$ (表 1 , Entry 6); 同时，反应混合物中还有大量原料 $N$-烯丙基 咔唑-3-甲醛未反应，即乙腈为溶剂不利于中间产物双 席夫碱的生成. 另外, 在双席夫碱重排生成苯并咪唑类 化合物的过程中需有活泼氢的参与 ${ }^{[22]}$, 故非质子化溶 剂乙腈也不利于该重排的进行. 因此, 乙腈为溶剂的反 应，2c 的产率远低于同等条件下甲醇为溶剂的反应. 如 将溶剂甲醇的用量增加至 15 或 $20 \mathrm{~mL}$, 则 $2 \mathrm{c}$ 产率相继 下降，而 $3 \mathbf{c}$ 产率相应增加，但总产率均底于表 1 , Entry 1 的 97.1\%(表 1, Entries 2, 3). 溶剂用量增加，反应物浓度 降低，不利于生成中间产物双席夫碱，故 2c 产率下降; 与此同时, 有利于生成经由单席夫碱-苯并咪唑啉中间 体所生成的产物 $\mathbf{3 c}$.

反应物的配比决定了产物的组成. 如表 1 中 Entry 1 所示, $N$-烯丙基咔唑-3-甲醛与邻苯二胺在等物质的量的 比为 2.2: 1 时反应，化合物 $2 \mathrm{c}$ 的产率远高于化合物 3c; 把两反应物的配比改为 $1: 1.2,45{ }^{\circ} \mathrm{C}$ 下将溶有 2.0 $\mathrm{mmol}$ 醛的 $8 \mathrm{~mL}$ 无水甲醇溶液缓慢滴加到 $2.4 \mathrm{mmol}$ 邻 苯二胺的 $2 \mathrm{~mL}$ 无水甲醇溶液中, $40 \mathrm{~min}$ 滴加毕, 保温

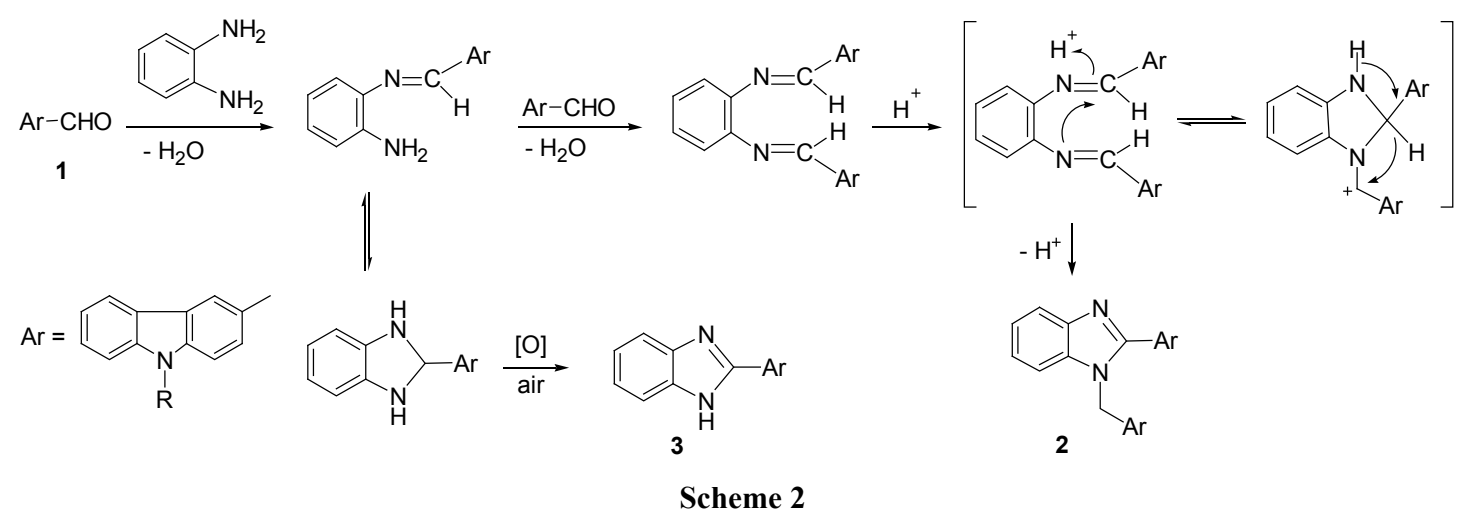

表 1 溶剂的选择及用量、反应温度和反应物配比对反应的影响

Table 1 Effects of selection \& amount of solvents, mole ratio of reagents, and temperatures on the reactions

\begin{tabular}{llcccc}
\hline Entry & Solvent & $n(\mathbf{1} \mathbf{c}): n$ (Diamine) & Temp. $/{ }^{\circ} \mathrm{C}$ & Yield $^{b} / \%$ of product 2c & Yield $^{b} / \%$ of product 3c \\
\hline 1 & Methanol $(10 \mathrm{~mL})$ & $2.2: 1$ & 45 & 78.6 & 18.5 \\
2 & Methanol $(15 \mathrm{~mL})$ & $2.2: 1$ & 45 & 69.2 & 24.6 \\
3 & Methanol $(20 \mathrm{~mL})$ & $2.2: 1$ & 45 & 53.0 & 32.1 \\
4 & Methanol $(10 \mathrm{~mL})$ & $2.2: 1$ & 65 & 71.7 & 24.3 \\
5 & Methanol $(10 \mathrm{~mL})$ & $1.0: 1.2$ & 45 & 34.6 & 55.7 \\
6 & Acetonitrile $(10 \mathrm{~mL})$ & $2.2: 1$ & 45 & 35.7 & 36.3 \\
\hline
\end{tabular}

${ }^{a}$ Reaction procedure: all Entries except Entry 5, 9-allyl-9H-carbazole-3-carbaldehyde (1c) dissolved in solvent, then the diamine was added; for Entry 5, diamine dissolved in methanol, then $\mathbf{1 c}$ in methanol was added dropwise. ${ }^{b}$ Isolated yield. ${ }^{c}$ Purified by recrystallization or column chromatography. 
反应 $6.5 \mathrm{~h}$, 所得化合物 $\mathbf{2 c}$ 产率为 $34.6 \%$, 化合物 $\mathbf{3 c}$ 产 率为 $55.7 \%$ (表 1, Entry 5). 邻苯二胺过量的情况下, 反 应有利于生成单席夫碱中间体及其环化产物化合物 $\mathbf{3 c}$, 但也生成了一定量的化合物 $\mathbf{2 c}$.

升高反应温度, 有利于生成化合物 $3 \mathrm{c}$ (表 1 , Entry 4). 反应中, 醛与邻苯二胺经缩合-环化反应生成中间 体苯并咪唑啉, 后者被空气中的 $\mathrm{O}_{2}$ 氧化生成化合物 3c, 升高温度有利于氧化脱氢反应.

\section{2 结论}

无催化剂存在下, 以无水甲醇为溶剂, $N$-烃基咔唑3-甲醛与邻苯二胺经缩合-环化 “一锅” 反应合成了两 类新型含咔唑结构单元的苯并咪唑衍生物 $\mathbf{2 a} \sim 2 \mathrm{e}$ 和 3a 3e. 合成反应中, 反应物配比、溶剂选择及其用量 对反应结果的影响较大, 在一定范围内温度对反应的影 响相对较小. 通过改变反应物的摩尔比可选择性地得到 以其中一类苯并咪唑衍生物为主的产物. 本合成方法具 有反应条件温和、操作简便、产率理想和对环境友好等 优点.

\section{3 实验部分}

\section{1 仪器与试剂}

X-6 显微熔点仪(北京泰克仪器有限公司, 温度未经 校正); Eqinox55 红外光谱仪(德国 Bruker 公司); Inova 300NB 核磁共振仪(美国 Varian 公司); 4000QTRAP 质谱 仪(美国 AB Sciex 公司); CHNS/O 2400 有机元素分析仪 (美国 Perkin Elmer 公司).

所用试剂均为市售分析纯或化学纯试剂, 未进一步 纯化; 实验中所用水为去离子水.

\section{2 实验方法}

\subsubsection{N-烃基咔唑-3-甲醛的合成}

$N$-烃基咔唑-3-甲醛(1)的合成方法见辅助材料.

\subsection{2 含咔唑基苯并咪唑衍生物 2 和 3 的合成}

合成通法: $25 \mathrm{~mL}$ 二口瓶中加入 $2.2 \mathrm{mmol} \mathrm{N}$-烃基咔 唑-3-甲醛(1), $10 \mathrm{~mL}$ 无水甲醇, 升温至 $45{ }^{\circ} \mathrm{C}$ 使固体完 全溶解, 加入 $1.0 \mathrm{mmol}$ 邻苯二胺, 保持温度 $45{ }^{\circ} \mathrm{C}$, 磁 力搅拌下反应一定时间(TLC 跟踪反应). 反应毕, 得黄 色沉淀, 滤集固体, 以无水甲醇重结晶, 过滤, 得化合 物 2; 重结晶母液经硅胶柱层析分离, 乙酸乙酯洗脱, 得化合物 3. 其中合成 $3 \mathbf{a} \sim \mathbf{3 c}$ 反应 $7 \mathrm{~h}$, 合成 $\mathbf{3 d}$ 和 $\mathbf{3 e}$ 反应 $6 \mathrm{~h}$.

1-(9-乙基咔唑-3-亚甲基)-2-(9-乙基咔唑-3-基)苯并 咪唑(2a): 浅黄色固体, 产率 $73.4 \%$. m.p. 153.3 154.1 ${ }^{\circ} \mathrm{C} ;{ }^{1} \mathrm{H}$ NMR $\left(\mathrm{CDCl}_{3}, 300 \mathrm{MHz}\right) \delta: 8.53(\mathrm{~d}, J=1.2 \mathrm{~Hz}, 1 \mathrm{H}$,
ArH), $8.02 \sim 7.87(\mathrm{~m}, 5 \mathrm{H}, \operatorname{ArH}), 7.51 \sim 7.13(\mathrm{~m}, 12 \mathrm{H}$, $\operatorname{ArH}), 5.72\left(\mathrm{~s}, 2 \mathrm{H}, \mathrm{CH}_{2}\right), 4.39 \sim 4.34\left(\mathrm{~m}, 4 \mathrm{H}, \mathrm{CH}_{2}-\mathrm{Me}\right)$, $1.49 \sim 1.43\left(\mathrm{~m}, 6 \mathrm{H}, \mathrm{CH}_{3}\right)$; IR ( $\left.\mathrm{KBr}\right)$ v: 3049, 1631, 1601, 1491, 1455, 1440, 1345, 1331, 748, $732 \mathrm{~cm}^{-1}$; MS (ESI) $m / z: 519.5(\mathrm{M}+\mathrm{H})^{+}$. Anal. calcd for $\mathrm{C}_{36} \mathrm{H}_{30} \mathrm{~N}_{4}: \mathrm{C} 83.37, \mathrm{H}$ 5.83, N 10.80; found C 83.18, H 5.91, N 10.90 .

9-乙基-3-(苯并咪唑-2-基)咔唑(3a): 浅棕色固体, 产率 20.4\%. m.p. 283.9 285.8 ${ }^{\circ} \mathrm{C} ;{ }^{1} \mathrm{H}$ NMR (DMSO- $d_{6}$, $300 \mathrm{MHz}) \delta: 12.84(\mathrm{~d}, J=0.9 \mathrm{~Hz}, 1 \mathrm{H}, \mathrm{NH}), 8.97$ (d, $J=$ $1.2 \mathrm{~Hz}, 1 \mathrm{H}, \mathrm{ArH}), 8.30 \sim 8.20(\mathrm{~m}, 2 \mathrm{H}, \mathrm{ArH}), 7.76$ (d, $J=$ $8.7 \mathrm{~Hz}, 1 \mathrm{H}, \mathrm{ArH}), 7.64$ (d, $J=8.4 \mathrm{~Hz}, 1 \mathrm{H}, \mathrm{ArH}), 7.58$ (dd, $J=5.9,3.2 \mathrm{~Hz}, 2 \mathrm{H}, \mathrm{ArH}), 7.49(\mathrm{t}, J=7.6 \mathrm{~Hz}, 1 \mathrm{H}, \mathrm{ArH})$, $7.26(\mathrm{t}, J=7.5 \mathrm{~Hz}, 1 \mathrm{H}, \mathrm{ArH}), 7.28 \sim 7.14(\mathrm{~m}, 2 \mathrm{H}, \mathrm{ArH})$, 4.49 (q, $\left.J=7.1 \mathrm{~Hz}, 2 \mathrm{H}, \mathrm{CH}_{2}\right), 1.35(\mathrm{t}, J=7.1 \mathrm{~Hz}, 3 \mathrm{H}$, $\mathrm{CH}_{3}$ ); IR (KBr) v: 3049, 1622, 1600, 1471, 1441, 1407, 1370, 1346, 750, $731 \mathrm{~cm}^{-1}$; MS (ESI) $m / z: 312.4(\mathrm{M}+$ $\mathrm{H})^{+}$. Anal. calcd for $\mathrm{C}_{21} \mathrm{H}_{17} \mathrm{~N}_{3}$ : C 81.00, H 5.50, N 13.49; found $\mathrm{C} 81.13, \mathrm{H} 5.45, \mathrm{~N} 13.40$.

1-(9-异丙基咔唑-3-亚甲基)-2-(9-异丙基咔唑-3-基) 苯并咪唑(2b): 浅黄色固体, 产率 60.2\%. m.p. 141.0 $142.2{ }^{\circ} \mathrm{C} ;{ }^{1} \mathrm{H}$ NMR $\left(\mathrm{CDCl}_{3}, 300 \mathrm{MHz}\right) \delta: 8.54(\mathrm{~d}, J=1.5$ $\mathrm{Hz}, 1 \mathrm{H}, \mathrm{ArH}), 8.01 \sim 7.84(\mathrm{~m}, 5 \mathrm{H}, \mathrm{ArH}), 7.54 \sim 7.12(\mathrm{~m}$, $12 \mathrm{H}, \mathrm{ArH}), 5.72\left(\mathrm{~s}, 2 \mathrm{H}, \mathrm{CH}_{2}\right), 5.04 \sim 4.94(\mathrm{~m}, 2 \mathrm{H}, \mathrm{CH})$, $1.73\left(\mathrm{~d}, J=6.9 \mathrm{~Hz}, 6 \mathrm{H}, \mathrm{CH}_{3}\right), 1.71(\mathrm{~d}, J=6.9 \mathrm{~Hz}, 6 \mathrm{H}$, $\mathrm{CH}_{3}$ ); IR (KBr) v: 3050, 1626, 1599, 1489, 1456, 1422, 1385, 1380, 747, $731 \mathrm{~cm}^{-1}$; MS (ESI) $\mathrm{m} / z: 547.6(\mathrm{M}+$ $\mathrm{H})^{+}$. Anal. calcd for $\mathrm{C}_{38} \mathrm{H}_{34} \mathrm{~N}_{4}$ : C 83.48, H 6.27, N 10.25; found $\mathrm{C} 83.65, \mathrm{H} 6.16, \mathrm{~N} 10.18$.

9-异丙基-3-(苯并咪唑-2-基)咔唑(3b): 浅棕色固体, 产率 29.1\%. m.p. 289.5 290.9 ${ }^{\circ} \mathrm{C}$; ${ }^{1} \mathrm{H}$ NMR (DMSO- $d_{6}$, $300 \mathrm{MHz}) \delta: 12.84$ (s, 1H, NH), 8.97 (d, $J=1.2 \mathrm{~Hz}, 1 \mathrm{H}$, ArH), $8.28 \sim 8.20(\mathrm{~m}, 2 \mathrm{H}, \mathrm{ArH}), 7.85(\mathrm{~d}, J=8.7 \mathrm{~Hz}, 1 \mathrm{H}$, $\mathrm{ArH}), 7.72(\mathrm{~d}, J=8.4 \mathrm{~Hz}, 1 \mathrm{H}, \mathrm{ArH}), 7.59$ (dd, $J=5.9,3.2$ $\mathrm{Hz}, 2 \mathrm{H}, \mathrm{ArH}), 7.45$ (t, $J=7.8 \mathrm{~Hz}, 1 \mathrm{H}, \mathrm{ArH}), 7.24$ (t, $J=7.4$ $\mathrm{Hz}, 1 \mathrm{H}, \mathrm{ArH}), 7.20 \sim 7.14(\mathrm{~m}, 2 \mathrm{H}, \mathrm{ArH}), 5.22 \sim 5.08(\mathrm{~m}$, $1 \mathrm{H}, \mathrm{CH}), 1.65$ (d, $\left.J=6.9 \mathrm{~Hz}, 6 \mathrm{H}, \mathrm{CH}_{3}\right)$; IR (KBr) $v: 3049$, 1629, 1600, 1471, 1439, 1403, 1369, 1340, $747 \mathrm{~cm}^{-1}$; MS (ESI) $m / z$ : $326.3(\mathrm{M}+\mathrm{H})^{+}$. Anal. calcd for $\mathrm{C}_{22} \mathrm{H}_{19} \mathrm{~N}_{3}$ : C 81.20, H 5.89, N 12.91; found C 81.43, H 5.76, N 12.82.

1-(9-烯丙基咔唑-3-亚甲基)-2-(9-烯丙基咔唑-3-基) 苯并咪唑 $(2 \mathrm{c})$ : 浅黄色固体, 产率 78.6\%. m.p. 143.5 $144.8{ }^{\circ} \mathrm{C} ;{ }^{1} \mathrm{H}$ NMR $\left(\mathrm{CDCl}_{3}, 300 \mathrm{MHz}\right) \delta: 8.51$ (d, $J=1.5$ $\mathrm{Hz}, 1 \mathrm{H}, \mathrm{ArH}), 8.00 \sim 7.85$ (m, 5H, ArH), 7.49 7.13 (m, $12 \mathrm{H}, \mathrm{ArH}), 6.07 \sim 5.92(\mathrm{~m}, 2 \mathrm{H}, \mathrm{CH}=), 5.72\left(\mathrm{~s}, 2 \mathrm{H}, \mathrm{CH}_{2}\right)$, $5.19\left(\mathrm{~d}, J=10.2, \mathrm{~Hz}, 1 \mathrm{H}, \mathrm{CH}=\mathrm{CH}_{2}\right), 5.17(\mathrm{~d}, J=10.2 \mathrm{~Hz}$, 
$\left.1 \mathrm{H}, \mathrm{CH}=\mathrm{CH}_{2}\right), 5.07\left(\mathrm{~d}, J=17.1, \mathrm{~Hz}, 1 \mathrm{H}, \mathrm{CH}=\mathrm{CH}_{2}\right)$, $5.04\left(\mathrm{~d}, J=17.1 \mathrm{~Hz}, 1 \mathrm{H}, \mathrm{CH}=\mathrm{CH}_{2}\right), 4.92(\mathrm{~d}, J=6.0 \mathrm{~Hz}$, $4 \mathrm{H}, \mathrm{NCH}_{2}$ ); IR (KBr) v: 3047, 1629, 1602, 1491, 1455, 1435, 1346, 1330, 746, $731 \mathrm{~cm}^{-1}$; MS (ESI) $\mathrm{m} / \mathrm{z}: 543.5$ $(\mathrm{M}+\mathrm{H})^{+}$. Anal. calcd for $\mathrm{C}_{38} \mathrm{H}_{30} \mathrm{~N}_{4}$ : C 84.10, H 5.57, N 10.32; found C 84.02, H 5.61, N 10.40 .

9-烯丙基-3-(苯并咪唑-2-基)咔唑(3c): 浅棕色固体, 产率 18.5\% m.p. 235.6 236.7 ${ }^{\circ} \mathrm{C} ;{ }^{1} \mathrm{H}$ NMR (DMSO- $d_{6}$, $300 \mathrm{MHz}) \delta: 12.83(\mathrm{~d}, J=0.9 \mathrm{~Hz}, 1 \mathrm{H}, \mathrm{NH}), 8.98(\mathrm{~d}, J=$ $1.2 \mathrm{~Hz}, 1 \mathrm{H}, \mathrm{ArH}), 8.29 \sim 8.22(\mathrm{~m}, 2 \mathrm{H}, \mathrm{ArH}), 7.72(\mathrm{~d}, J=$ $8.7 \mathrm{~Hz}, 1 \mathrm{H}, \mathrm{ArH}), 7.62 \sim 7.52(\mathrm{~m}, 3 \mathrm{H}, \mathrm{ArH}), 7.48(\mathrm{t}, J=$ $7.6 \mathrm{~Hz}, 1 \mathrm{H}, \mathrm{ArH}), 7.27$ (t, $J=7.4 \mathrm{~Hz}, 1 \mathrm{H}, \mathrm{ArH}), 7.20 \sim$ $7.14(\mathrm{~m}, 2 \mathrm{H}, \mathrm{ArH}), 6.07 \sim 5.95(\mathrm{~m}, 1 \mathrm{H}, \mathrm{CH}=), 5.12(\mathrm{~d}$, $\left.J=12.6 \mathrm{~Hz}, 1 \mathrm{H},=\mathrm{CH}_{2}\right), 5.11(\mathrm{~d}, J=16.5 \mathrm{~Hz}, 1 \mathrm{H}$, $\left.=\mathrm{CH}_{2}\right), 5.00\left(\mathrm{~d}, J=17.1 \mathrm{~Hz}, 1 \mathrm{H}, \mathrm{CH}_{2}\right), 4.98(\mathrm{~d}, J=17.1$ $\mathrm{Hz}, 1 \mathrm{H}, \mathrm{CH}_{2}$ ); IR (KBr) v: 3050, 1630, 1602, 1472, 1448, 1418, 1404, 1374, 1318, $747 \mathrm{~cm}^{-1}$; MS (ESI) $\mathrm{m} / \mathrm{z}: 324.4$ $(\mathrm{M}+\mathrm{H})^{+}$. Anal. calcd for $\mathrm{C}_{22} \mathrm{H}_{17} \mathrm{~N}_{3}$ : C 81.71, H 5.30, N 12.99; found C 81.60, H 5.35, N 13.06 .

1-(9-正丁基咔唑-3-亚甲基)-2-(9-正丁基咔唑-3-基) 苯并咪唑(2d): 浅黄色固体, 产率 77.1\%. m.p. 102.4 $103.6{ }^{\circ} \mathrm{C} ;{ }^{1} \mathrm{H}$ NMR $\left(\mathrm{CDCl}_{3}, 300 \mathrm{MHz}\right) \delta$ : 8.51 (d, $J=1.2$ $\mathrm{Hz}, 1 \mathrm{H}, \mathrm{ArH}), 8.00 \sim 7.87(\mathrm{~m}, 5 \mathrm{H}, \mathrm{ArH}), 7.50 \sim 7.11(\mathrm{~m}$, $12 \mathrm{H}, \mathrm{ArH}), 5.72\left(\mathrm{~s}, 2 \mathrm{H}, \mathrm{CH}_{2}\right), 4.32(\mathrm{t}, J=7.2 \mathrm{~Hz}, 4 \mathrm{H}$, $\left.\mathrm{CH}_{2}\right), 1.93 \sim 1.85\left(\mathrm{~m}, 4 \mathrm{H}, \mathrm{CH}_{2}\right), 1.47 \sim 1.37(\mathrm{~m}, 4 \mathrm{H}$, $\left.\mathrm{CH}_{2}-\mathrm{Me}\right), 0.97$ (t, $\left.J=7.2 \mathrm{~Hz}, 3 \mathrm{H}, \mathrm{CH}_{3}\right), 0.95$ (t, $J=7.2 \mathrm{~Hz}$, $\left.3 \mathrm{H}, \mathrm{CH}_{3}\right)$; IR (KBr) v: 3052, 1630, 1601, 1491, 1456, 1439, 1347, 1331, 748, $731 \mathrm{~cm}^{-1}$; MS (ESI) $\mathrm{m} / \mathrm{z}: 575.6$ $(\mathrm{M}+\mathrm{H})^{+}$. Anal. calcd for $\mathrm{C}_{40} \mathrm{H}_{38} \mathrm{~N}_{4}$ : C 83.59, H 6.66, N 9.75; found C 83.49, H 6.71, N 9.81.

9-正丁基-3-(苯并咪唑-2-基)咔唑(3d)：浅棕色固体, 产率 19.1\%. m.p. 290.3 291.5 ${ }^{\circ} \mathrm{C} ;{ }^{1} \mathrm{H}$ NMR (DMSO- $d_{6}$, $300 \mathrm{MHz}) \delta: 12.82(\mathrm{~s}, 1 \mathrm{H}, \mathrm{NH}), 8.97(\mathrm{~d}, J=1.2 \mathrm{~Hz}, 1 \mathrm{H}$, ArH), $8.30 \sim 8.20(\mathrm{~m}, 2 \mathrm{H}, \operatorname{ArH}), 7.75(\mathrm{~d}, J=8.7 \mathrm{~Hz}, 1 \mathrm{H}$, ArH), $7.64 \sim 7.52(\mathrm{~m}, 3 \mathrm{H}, \mathrm{ArH}), 7.48(\mathrm{t}, J=7.7 \mathrm{~Hz}, 1 \mathrm{H}$, $\operatorname{ArH}), 7.25(\mathrm{t}, J=7.3 \mathrm{~Hz}, 1 \mathrm{H}, \mathrm{ArH}), 7.20 \sim 7.14(\mathrm{~m}, 2 \mathrm{H}$, $\operatorname{ArH}), 4.43\left(\mathrm{t}, J=7.0 \mathrm{~Hz}, 2 \mathrm{H}, \mathrm{CH}_{2}\right), 1.82 \sim 1.72(\mathrm{~m}, 2 \mathrm{H}$, $\left.\mathrm{CH}_{2}\right), 1.38 \sim 1.22\left(\mathrm{~m}, 2 \mathrm{H}, \mathrm{CH}_{2}\right), 0.88(\mathrm{t}, J=7.3 \mathrm{~Hz}, 3 \mathrm{H}$, $\mathrm{CH}_{3}$ ); IR (KBr) v: 3047, 1629, 1600, 1467, 1442, 1401, 1367, 1351, 748, $734 \mathrm{~cm}^{-1}$; MS (ESI) m/z: $340.5(\mathrm{M}+$ $\mathrm{H})^{+}$. Anal. calcd for $\mathrm{C}_{23} \mathrm{H}_{21} \mathrm{~N}_{3}$ : C 81.39, H 6.24, N 12.38; found $\mathrm{C} 81.53, \mathrm{H} 6.15, \mathrm{~N} 12.22$.

1-(9-正己基咔唑-3-亚甲基)-2-(9-正己基咔唑-3-基) 苯并咪唑 $(2 \mathrm{e})$ : 浅黄色固体, 产率 78.5\%. m.p. 91.5 $92.6{ }^{\circ} \mathrm{C} ;{ }^{1} \mathrm{H} \mathrm{NMR}\left(\mathrm{CDCl}_{3}, 300 \mathrm{MHz}\right) \delta: 8.57$ (s, $\left.1 \mathrm{H}, \mathrm{ArH}\right)$,
$8.02 \sim 7.90(\mathrm{~m}, 5 \mathrm{H}, \mathrm{ArH}), 7.52 \sim 7.15(\mathrm{~m}, 12 \mathrm{H}, \mathrm{ArH}), 5.71$ $\left(\mathrm{s}, 2 \mathrm{H}, \mathrm{CH}_{2}\right), 4.29\left(\mathrm{t}, J=6.9 \mathrm{~Hz}, 4 \mathrm{H}, \mathrm{CH}_{2}\right), 1.94 \sim 1.83(\mathrm{~m}$, $\left.4 \mathrm{H}, \mathrm{CH}_{2}\right), 1.42 \sim 1.27\left(\mathrm{~m}, 12 \mathrm{H}, \mathrm{CH}_{2}\right), 0.91(\mathrm{t}, J=6.6 \mathrm{~Hz}$, $\left.3 \mathrm{H}, \mathrm{CH}_{3}\right), 0.89\left(\mathrm{t}, J=7.2 \mathrm{~Hz}, 3 \mathrm{H}, \mathrm{CH}_{3}\right)$; IR (KBr) v: 3053, 1630, 1601, 1491, 1456, 1444, 1346, 1332, 746, $733 \mathrm{~cm}^{-1}$; MS (ESI) $m / z$ : $631.7(\mathrm{M}+\mathrm{H})^{+}$. Anal. calcd for $\mathrm{C}_{44} \mathrm{H}_{46} \mathrm{~N}_{4}$ : C 83.77, H 7.35, N 8.88; found C 84.00, H 7.23, N 8.78.

9-正己基-3-(苯并咪唑-2-基)咔唑(3e)：浅棕色固体, 产率 15.3\%. m.p. 269.7 270.9 ${ }^{\circ} \mathrm{C} ;{ }^{1} \mathrm{H}$ NMR (DMSO- $d_{6}$, $300 \mathrm{MHz}) \delta: 12.82(\mathrm{~d}, J=1.2 \mathrm{~Hz}, 1 \mathrm{H}, \mathrm{NH}), 8.97$ (d, $J=$ $1.2 \mathrm{~Hz}, 1 \mathrm{H}, \mathrm{ArH}), 8.30 \sim 8.20(\mathrm{~m}, 2 \mathrm{H}, \mathrm{ArH}), 7.74$ (d, $J=$ $8.7 \mathrm{~Hz}, 1 \mathrm{H}, \mathrm{ArH}), 7.65 \sim 7.53(\mathrm{~m}, 3 \mathrm{H}, \mathrm{ArH}), 7.47(\mathrm{t}, J=$ $7.6 \mathrm{~Hz}, 1 \mathrm{H}, \mathrm{ArH}), 7.25$ (t, $J=7.5 \mathrm{~Hz}, 1 \mathrm{H}, \mathrm{ArH}), 7.20 \sim$ $7.14(\mathrm{~m}, 2 \mathrm{H}, \mathrm{ArH}), 4.41\left(\mathrm{t}, J=6.9 \mathrm{~Hz}, 2 \mathrm{H}, \mathrm{CH}_{2}\right), 1.82 \sim$ $1.72\left(\mathrm{~m}, 2 \mathrm{H}, \mathrm{CH}_{2}\right), 1.28 \sim 1.13\left(\mathrm{~m}, 6 \mathrm{H}, \mathrm{CH}_{2}\right), 0.78(\mathrm{t}, J=$ $\left.6.9 \mathrm{~Hz}, 3 \mathrm{H}, \mathrm{CH}_{3}\right)$; IR (KBr) v: 3049, 1625, 1600, 1467, 1442, 1406, 1369, 1351, 748, $735 \mathrm{~cm}^{-1}$; MS (ESI) m/z: $368.5(\mathrm{M}+\mathrm{H})^{+}$. Anal. calcd for $\mathrm{C}_{25} \mathrm{H}_{25} \mathrm{~N}_{3}: \mathrm{C} 81.71, \mathrm{H}$ 6.86, N 11.43; found C 81.60, H 6.91, N 11.50.

辅助材料(Supporting Information) 提供了 $N$-烃基咔 唑-3-甲醛(化合物 1)的合成方法、标题化合物的 MS、IR 和 ${ }^{1} \mathrm{H}$ NMR 数据以及 ${ }^{1} \mathrm{H}$ NMR 谱图. 这些材料可以免费 从本刊网站(http://sioc-journal.cn/)上下载.

\section{References}

[1] Singh, M.; Tandon, V. Eur. J. Med. Chem. 2011, 46, 659.

[2] Zhan, P.; Chen, X. W.; Li, D. Y.; Fang, Z. J.; De Clercq, E.; Liu, X. Y. Med. Chem. Comm. 2013, 33, S1, E1.

[3] Sharma, M. C.; Kohli, D.; Sharma, S.; Sharma, A. D. Pharma Sin. 2010, $1,104$.

[4] Takahashi, K.; Hashimoto, N.; Nakama, C.; Kamata, K.; Sasaki, K.; Yoshimoto, R.; Ohyama, S.; Hosaka, H.; Maruki, H.; Nagata, Y.; Eiki, J.; Nishimura, T. Bioorg. Med. Chem. 2009, 17, 7042.

[5] Meng, J.-P.; Geng, R.-X.; Zhou, C.-H.; Gan, L.-L. Chin. J. New Drugs 2009, 18, 1505 (in Chinese).

(孟江平, 耿蓉霞, 周成合, 甘淋玲, 中国新药杂志, 2009, 18 , 1505.)

[6] Peng, P.; Xiong, J.-F.; Li, B.; Mo, G.-Z.; Chen, R.-H.; Wang, Z.-Y. Chin. J. Org. Chem. 2013, 33, 1891 (in Chinese). (彭看, 熊金锋, 李豹, 莫广珍, 陈任宏, 汪朝阳, 有机化学, 2013, 33, 1891.)

[7] Batista, R. M. F.; Costa, S. P. G.; Raposo, M. M. M. J. Photochem. Photobiol., A: Chem. 2013, 259, 33.

[8] Park, S.; Kwon, O. H.; Kim, S.; Park, S.; Choi, M. G.; Cha, M.; Park, S. Y.; Jang, D. J. J. Am Chem. Sci. 2005, 127, 10070.

[9] Zhang, R.; Li, J.-T.; Fu, C.-L.; Luo, X.-T. Mater. Rev. 2011, 25, 61 (in Chinese).

(张蓉, 李锦堂, 傅翠梨, 罗学涛, 材料导报, 2011, 25, 61.)

[10] Wu Y.-F.; Cui, Y.-N.; Li, S.-M.; Jia, Y.-P.; Yin, J.-M. Chin. J. Inorg. Chem. 2012, 28, 910 (in Chinese).

(吴玉防, 崔颖娜, 李慎敏, 贾颖萍, 尹静梅, 无机化学学报, 
2012, 28,910.)

[11] Pina, J.; De Melo, J. S. S.; Batista, R. M. F.; Costa, S. P. G.; Raposo, M. M. M. J. Org. Chem. 2013, 78, 11389.

[12] Liu, L-T.; Ishida, N.; Ashida, S.; Murakami, M. Org. Lett. 2011, $13,1666$.

[13] Yu, K.; Guan, S.-X.; Zhang, H.-W.; Zhou, B.-B.; Li, L. Nat. Sci. J. Harbin Normal Univ. 2006, 22, 70 (in Chinese).

(于凯, 关淑霞, 张宏伟, 周百斌, 李玲, 哈尔滨师范大学自然科 学学报, 2006, 22, 70.)

[14] Huang, B.; Tang, J.-N.; Jiang, W.; Yang, W.; Ban, X.-X.; Sun, Y.-M. Chin. J. Org. Chem. 2013, 33, 1395 (in Chinese). (黄斌, 唐雯楠, 蒋伟, 杨文, 班金金金鍂, 孙岳明, 有机化学, 2013, 33, 1395.)

[15] Boufatah, N.; Gellis, A.; Maldonado, J.; Vanelle, P. Tetrahedron 2004, 60, 9131.

[16] Lu, J.; Ge, H.-G.; Bai, Y.-J. Chin. J. Org. Chem. 2002, 22, 782 (in Chinese).

(路军, 葛红光, 白银娟, 有机化学, 2002, 22, 782.)

[17] Lee, K. J.; Janda, K. D. Can. J. Chem. 2001, 79, 1556.
[18] Das, B.; Harish, H.; Srinivas, Y. Tetrahedron Lett. 2007, 48, 61.

[19] Chari, M. A.; Shobha, D.; Sasaki, T. Tetrahedron Lett. 2011, 52, 5575 .

[20] Bahrami, K.; Khodaei, M. M.; Naali, F. J. Org. Chem. 2008, 73, 6835.

[21] Chakrabarty, M.; Mukherjee, R.; Karmakar, S.; Harigaya, Y. Monatsh. Chem. 2007, 138, 1279.

[22] Varala, R.; Nasreen, A.; Ramu, E.; Adapa, S. R. Tetrahedron Lett. 2007, 48, 69

[23] Yang, H.-W.; Yue, F.; Feng, S.; Wang, J.-D.; Liu, A.-H.; Chen, H.-M.; Yu, K.-B. Chin. J. Org. Chem. 2004, 24, 792 (in Chinese). (杨红伟, 岳凡, 封顺, 王吉德, 刘爱华, 陈华梅, 郁开北, 有机 化学, 2004, 24, 792.)

[24] Lin, S.-N.; Yang, L.-H. Tetrahedron Lett. 2005, 46, 4315.

[25] Cui, L.-J.; Xiao, S.-Y.; Yang, H.-S.; Xu, P.; Cheng, F.-S.; Li, Z.-H.; Liang, R.-H.; Xia, Z.-N. Chin. J. Org. Chem. 2011, 31, 672 (in Chinese).

(崔丽君, 肖尚友, 杨昊书, 徐盼, 程凡圣, 李正华, 梁荣辉, 夏 之宁, 有机化学, 2011, 31, 672.)

(Qin, X.) 